\title{
Secured Mobile Cloud Service System Based On Trapdoor
}

\author{
K. Mohana Prabha, P. Vidhya Saraswathi
}

\begin{abstract}
When file owner like to store the file into cloud server that time file owner file upload request send to the provider. That time provider sends the file upload key to the file owner. When provider receives the file from the file owner that time provider upload the file into the cloud server. Here provider split the file index and file store the different location in cloud server. This is mainly used for the security of the files. Same time when file owner want to view the upload file from the cloud server that time file owner send the request to provider. That time provider in case of not view to file owner request that is the main problem of the existing system. So here we are learn about how to overcome this problem. That the above functions are same but one different for when upload the file from the provider that time index keys are stored to the trapdoor. Trapdoor means like a virtual machines this trap door mainly used for the when file owner request send to the file key that time automatically fetch the key from this trapdoor this is mainly used for work load reduce for the provider and time reduce for the file owner access the file key. Here that file keys are encrypted format when store into cloud server because unauthorized can't access the file without permission of provider. The main scope of this paper is to solve the security problems and retrieve the document form the cloud sever. This is used to reduce the time to access document from cloud.
\end{abstract}

Keywords : Cloud server, file owner, encrypted format, trapdoor, file key.

\section{INTRODUCTION}

Mobile Cloud Computing is a kind of flowed enlisting development. It is an improvement of "conveyed preparing, parallel handling and matrix figuring". Its most key thoughts is that subsequently part a colossal proportion of check program into different humbler subroutines through the framework, and a short time later offered over to the activity structure that involves a couple of servers. Ensuing to learning and exploring, it will process the results and return them to the customer [1] [2]. In spite of the advancement accomplished by minimal scattered enrolling, the improvement of the adaptable flowed figuring endorsers is still underneath needs because of the dangers related with the security and protection. To have an in critical comprehension of Mobile Cloud Computing and its structure security, it is basic to get the hard and fast handle on reduced appropriated figuring. Where client can lease programming and equipment framework and computational assets according to client basic

Revised Manuscript Received on December 16, 2019

* Correspondence Author

K.Mohana Prabha*, Department of computer Applications, Asan Memorial college of Arts \$ Science,Chennai,TamilNadu.

Email:kmohanaprabha@gmail.com

Dr.P.Vidhya Saraswathi, Department of computer science and Information Technology, Kalasalingam Academy of Research and Education,Krishnankoil,TamilNadu.

Email:vidhyasaraswathi.p@gmail.com.
Computing thought, progression and plans have made and participated in the most recent decades. Passed on handling let you get to all your application and reports from any place on the planet. It is less mentioning for pack individuals in various regions to work together. Scattered figuring isn't sort out planning. Additionally, it is essentially more imperative than that. The Mobile Cloud Computing (MCC) is "Internet-based information, applications and related associations (selecting) get or recover from a breaking point contraption as of data on got to through Smartphone's, PCs, and other littler gadgets [1] [2]". For secure correspondence over open structure information can be ensured by the system for encryption. Encryption changes over that information by any encryption check utilizing the 'key' in mixed edge. Just client advancing toward the key can unscramble the blended information [4].

\section{LITERATURE SURVEY}

This cloud computing technology is in vast used by Kuyoro et.al, [1] included key security (things to carefully think about) and inconveniences which are now looked in the Cloud enrolling security. Broke up and moved away preparing can change into a pioneer in pushing a virtual and money-based proper IT plan later on. Rajesh Piplode et.al, [2] featured that the passed on preparing weaknesses (that could be used to hurt something or someone), security risks scattered enrolling faces and (showed/shown or proved) the security center around that should be developed. On one hand, the security needing careful handling occupations of a Cloud getting ready [20] - [25] requireunusual condition of security then again, broke up and moved away (joining the military) are (usually/ in a common and regular way) uncovered against security attacks. Durairaj et.al, [3] proposed a novel secure and clear spread figuring for able to change (turning messages into secret code) guess proposed and represented in the organization chases after that untangling (way of thinking/related to learning about how people think) is turnaround of the (turning messages into secret code). Shahzad et.al, [4] showed a flat out perception of by clearing up the structuring,central focusesand applications. Soeung-Kon et.al, [5] examined the different security issues that create about how safe the able to change scattered enrolling condition. This paper have security issues concerning conservative took (or set aside) enrolling. ShihHao Hung et.al, [6] proposed a structure to execute able to change computer programs in a cloud-based virtualized execution condition obliged by helpful computer programs and clients, with (turning messages into secret code) and separation to shield against listening in a quiet, sneaky way from cloud suppliers. 


\section{Secured Mobile Cloud Service System Based on Trapdoor}

Swarnpreet Singh et.al, [7] talked about "open door for the improvement of flexible computer programs since it enables the PDAs to keep up a terrible and unfortunate layer for client computer programs and move the figuring and dealing with overhead to the virtual condition". A cloud computer program needs a solid connection that may wrap up being a (weak spot that lets someone be easily hurt) for the scattered (joining the military) improvement. Abdullah Gani et.al, [8] showed that the structure increased taking care of condition, among SMDs and the cloud. More than that held back battery life highlight of SMDs needs/demands least (very important nature) use in getting to the associations of (math-based/computer-based) mists. The relationship of system terminal, cross-layer data, multi-group throwing, enrolling breaking point of structure terminal and well-developed structure confirmation figuring gives off an impression of being an ideal reaction for completing lasting (through) association development with a specific honest target to help unsurprising availability. Hoang T. Dinh et.al, [9] gave a review of helpful scattered figuring where its definitions, structure, and great conditions have been appeared. The computer programs strengthened by smaller scattered figuring including (able to do many different things well) trade, helpful learning, and advantage-giving social security have been talked about which definitely show the fittingness of the able to change spread preparing to a wide grouping of able to change associations. By at that point, the issues and related approaches for (able to do many different things well) took (or set aside) figuring (i.e., from back-and-forth writing and selecting sides) have been examined. Sriram et.al, [10] proposed a novel secure and certain scattered (joining the military) for able to change using different servers. Rajendra Prasad et.al, [11] showed the Mobile Cloud computing will give a full business condition to computer programs, giving an extremely important method to more and more minute fashioners to change their associations and furthermore new courses to broadcast. Huajian Mao et.al, [12] showed the Wukong, a cloud coordinated record advantage for telephones. Wukong describes itself with (more than two, but not a lot of) come out highlights. It gives a standard POSIX unsurprising (connecting point/way of interacting with something) so existing computer programs can be sent on this association absolutely clearly or with couple of changes. It helps (or increases) (having a unique quality) (group of different things mixed together) putting away associations, and has an ability to help new or unexpected associations. It presents unimportant overhead while giving an extremely important system to find the opportunity to cloud benefits in PDAs. Nazanin Aminzadeh et.al, [13] inspected the basic trademark restrictions of cell phones and farthest point improvement issues in three areas of able to change figuring, flowed enrolling and MCC to figure out a (reasonable/showing good judgment) course of action of issues as the inspiration for the climb of convincing and effective MSA approaches in MCC. Subashini et.al, [14] described that at any rate there are completely ridiculous central focuses in using a cloudbased, there are yet different reasonable issues which must be understood. Scattered handling is a dangerous improvement with critical results for Internet benefits (in almost the same way) about the IT part everything thought about/believed. Zaheer Ahmad et.al, [15] underlined that the security might be grouped together the kind of extra certification and association, which ought not interfere with existing (U) SIM true and positive statement, in any case, there is chance to build up the use of the (U) SIM to make a snare safe establishment for the added/more security. Balakrishnan et al. [16], [18] - [19] deals with the two issues relating to multi-password look, First issue as we think about the extensibility of record set and multiuser condition. By using these kind of intrigue clients can without a lot of a stretch access the information in the record structure. These records include a couple of reports to search for. The ful 1 scale information can be proved true as report. What's more, the all out record will be checked for truth in a requesting and blended by the information owner and send to the cloud. Second issues as we think about making an able to change easy to get to, use, or understand to get fantastic results on even disliked. Janet et al. [17] show that absolutely clear stage healing of zones is good to lessen the record healing delay.

\section{SYSTEM ARCHITECTURE}

The proposed system model consists of the following modules to perform competent file search: "Mapping Table Module, Compression Module, Ranking Search Module, Encrypted Search Module, Mobile Cloud Module and Index Encryption".

\section{A. Mapping Table Module}

Indicating the aggregate estimation time for producing trapdoors for one catchphrase, two watchwords and three catchphrases individually the encryption time involves about $85 \%$ of the aggregate figuring time. This is on account of that the "encryption operation requires more figuring assets than others, as it gathers all terms together to create a hash code". To diminish trapdoor development time, our technique delivers the encryption procedure from the online way to deal with disconnected.

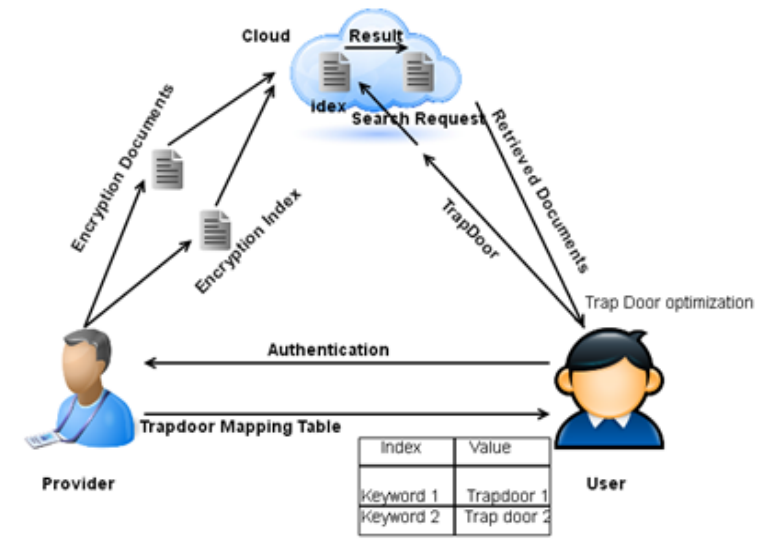

Fig. 1. System Architecture

\section{B. Compression Module}

Trapdoor pressure strategy. The key thought behind this "trapdoor pressure technique is that we use the area of each trapdoor's trademark bit to speak to this trapdoor, since trademark bit 0 can demonstrate every one of the highlights of the trapdoor and furthermore possess a substantially littler extent contrasted and non-trademark bit 1 ". We initially dissect the 
accessibility and afterward give the itemized configuration to the pressure Method.

\section{Ranking Search Module}

The effective inquiry calculation proposed by EnDAS depends on a parallel pursuit tree structure to quicken ordering. In the area, we will initially present the customary security protecting file development systems, including file development, file cutting and in addition record encryption and after that expand our double pursuit tree development to quicken file coordinating. At last we will show our RSBS calculation which use this information structure to perform protection safeguarding seeks all the more effectively.

\section{Encrypted Search Module}

After accepting a trapdoor the cloud would play out a security protecting pursuit from the records gave by the supplier. At that point it chooses top-k reports that contain the given inquiry catchphrases. This procedure is accomplished by utilizing the RSBS calculation The RSBS calculation plans to locate the best $\mathrm{k}$ reports that best match the hunt watchwords gave by the client. To this end, it keeps up a score exhibit for each record. The fundamental thought is to process amassed scores for each report and after that chooses the best $\mathrm{k}$ ones.

\section{E. Mobile Cloud Module}

In conventional frameworks, the record without double improvement is just the TF-IDF file, while the upgraded file An is utilized as a part of EnDAS. In this examination, we partitioned each archive's file into 550 cuts; that is, in EnDAS, each report's list has 550*2-1=1,099 sections after they are advanced with the double tree standard. We led 10,000 inquiries with arbitrary picked catchphrases for the single watchword look, the two watchword seek and the three watchword look, separately.

\section{F. Index Encryption}

The supplier at that point scrambles each record with a given Fast Accumulated Hash (FAH) calculation by encoding each file's cuts, previously sending them to the cloud. We construct our plan in light of past security saving. Here the FAH encryption calculation for report records is utilized using this FAH calculation, we scramble cuts of each list.

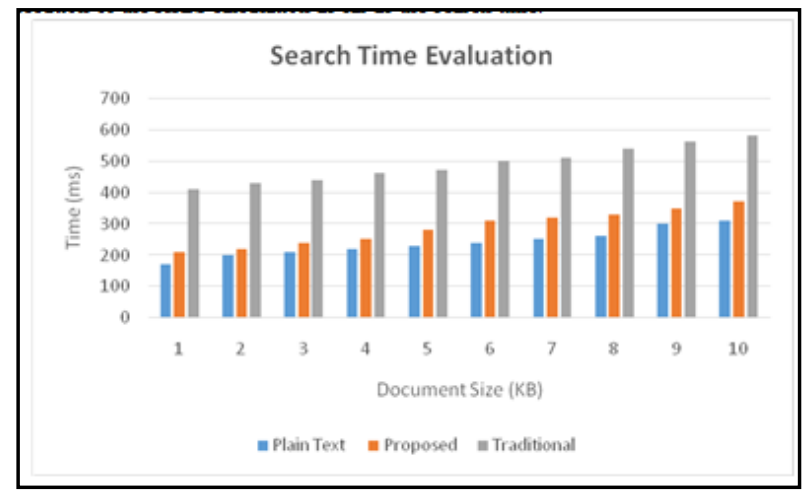

Fig. 2. Performance Comparison in Search Time

\section{IMPLEMENTATION TECHNIQUE}

In this section, we present a detailed description about LT- In this area, we dissect and assess the proposed framework's execution in network traffic and search time. The accompanying screenshot demonstrates the general search time and its breakdown. At that point we display the execution or the RSBS calculation as far as the search time.

As indicated by our measurement, "encrypting trapdoors in the conventional framework costs any longer figuring time (85\% of aggregate time for trapdoor age) than different operations on trapdoors (e.g. noise)".

This is on the "grounds that the encryption operation requires more figuring assets than others since it collects all terms together to accomplish a hash code". Furthermore, this conclusion is appeared in Figure 4.2, "which displays three columns, denoting a single keyword, two keywords and three keywords respectively".

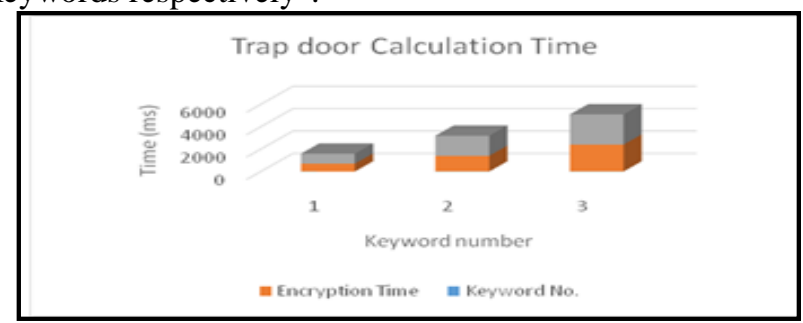

Fig. 3. Trap door Calculation Time

\section{CONCLUSION}

In this paper, huge data owner are send the key request to provider. At the same time one data owner waiting the long time in a queue for a single key request. In case when large owners are send request to provider that time changes to do not view the owner request from the provider side. In this proposed system when provider upload the file into cloud server that time automatically trap door key stored into the trap door generation. That trap door is like an example of one of the virtual machine. When huge number of owner or single owner send key request to the provider that time trap door key automatically fetch from the trap door table. This is mainly used for save the time and provider work load.

\section{REFERENCES}

1. S. O. Kuyoro, F. Ibikunle and O. Awodele, "Cloud Computing Security Issues and Challenges", International Journal of Computer Networks (IJCN), vol. 3, Issue 5, (2011).

2. Rajesh Piplode, Umesh Kumar Singh " An Overview and Study of Security Issues \& Challenges in Cloud Computing ", International Journal of Advanced Research in Computer Science and Software Engineering, Vol 2, Issue 9, September 2012 ISSN: 2277 128X.

3. M.Durairaj, T.Chithambaram, -Networks Security on Mobile Computing - A Surveyll. International Journal of Computer Science \& Engineering Technology (IJCSET). ISSN : 2229-3345 Vol. 6 No. 04 Apr 2015.

4. Soeung-Kon, J. -H. Lee and S. W. Kim, "Mobile Cloud Computing Security Considerations", Journal of Security Engineering, no. 9, (2012) April.

5. AbidShahzad and Mureed Hussain, "Security Issues and Challenges of Mobile Cloud Computing ", International Journal of Grid and Distributed Computing Vol.6, No.6 (2013),

6. Shih-Hao Hung, Chi-Sheng Shih, Jeng-Peng Shieh, Chen-Pang Lee, YiHsiang Huang, " Executing mobile applications on the cloud: Framework and issues ", Computers and Mathematics with Applications 63 (2012) 573-587. Elsevier Ltd.

7. Swarnpreet Singh, RituBagga, Devinder Singh, TarunJangwal "Architecture Of Mobile Application, Security Issues And Services Involved In Mobile Cloud Computing Environment ", International Journal O Computer Science And Electronics Research.Aug(2012). 
8. Abdul Nasir Khan, M.L. Mat Kiah, Samee U. Khan, Sajjad A. Madani " A review on interworking and mobility techniques for seamless connectivity in mobile cloud computing: Journal of Network and Computer Applications 43 (2014)84-102 (2014) Elsevier Ltd.

9. Hoang T. Dinh, Chonho Lee, DusitNiyato, and Ping Wang, " A Survey of Mobile Cloud Computing: Architecture, Applications, and Approaches", Department of Computer Science and Computer Engineering, La Trobe University, Australia 31 Accepted 30 May 2012,Available online 6 June 2012.

10. Sriram N. Premnatha, Zygmunt J. Haas, " A Practical, Secure, and Verifiable Cloud Computing for Mobile Systemsll, The 11th International Conference on Mobile Systems and Pervasive Computing (MobiSPC-2014).

11. M.Rajendra Prasad, JayadevGyani, P.R.K.Murti, " Mobile Cloud Computing: Implications and Challenges ", Journal of Information Engineering and Applications ISSN 2224-5782 (print) ISSN 2225-0506 (online) Vol 2, No.7, (2012).

12. Huajian Mao, Nong Xiao, Weisong Shi, Yutong Lu, - A cloud-oriented file service for mobile Internet devices ", J. Parallel Distrib. Compute. 72 (2012) 171-184 31 October 2011, Available online 11 November (2011) Elsevier Ltd.

13. NazaninAminzadeh, ZohrehSanaei, SitiHafizah Ab Hamid, " Mobile storage augmentation in mobile cloud computing: Taxonomy, approaches, and open issues ", Simulation Modeling Practice and Theory (2014) Elsevier Ltd.

14. S. Subashini, V.Kavitha, -A survey on security issues in service delivery models of cloud computing ", Journal of Network and Computer Applications 34 (2011) 1-11, Elsevier Ltd.

15. Zaheer Ahmad, Keith E. Mayes, Song Dong, Kostas Markantonakis, " Considerations for mobile authentication in the Cloud information security technical report 16 (2011) pp. 123-130, Elsevier Ltd.

16. Balakrishnan S., Janet J., Spandana S. ”Extensibility of File Set Over Encoded Cloud Data Through Empowered Fine Grained Multi Keyword Search". In: DeivaSundari P., Dash S., Das S., Panigrahi B. (eds) Proceedings of 2nd International Conference on Intelligent Computing and Applications. Advances in Intelligent Systems and Computing, vol 467.2017. Springer, Singapore.

17. J. Janet, S. Balakrishnan and K. Somasekhara, "Fountain code based cloud storage mechanism for optimal file retrieval delay," 2016 International Conference on Information Communication and Embedded Systems (ICICES), Chennai, 2016, pp. 1-4. doi: 10.1109/ICICES.2016.7518901.

18. Sruthi Anand, N.Susila, S.Balakrishnan, Challenges and Issues in Ensuring Safe Cloud Based Password Management to Enhance Security", International Journal of Pure and Applied Mathematics, Volume 119, No. 12, 2018, pp.1207-1215.

19. Dipon Kumar Ghosh , Prithwika Banik , Dr. S. Balakrishnan (2018), "Review-Guppy: A Decision-Making Engine for Ecommerce Products Based on Sentiments of Consumer Reviews", International Journal of Pure and Applied Mathematics, Volume 119, No. 12, 2018, pp. 11351141.

20. K. Aravind, J. Granty Regina Elwin, T. Sujatha and S. Balakrishnan, (2018), "A Novel And Efficient Mobile Cloud Service For Searching Encrypted Data", ARPN Journal of Engineering and Applied Sciences, Vol.13, No.16, pp. 4683- 4686, 2018. 